\title{
Efficacy and safety of immune checkpoint therapy in hepatocellular carcinoma: meta-analysis
}

\author{
Weina Tang", Long-Teng Ma", Yang Deng\#, Wei Wang, Hong-Wei Zhang \\ Department of Epidemiology, Second Military Medical University, Shanghai 200433, China. \\ \#These authors contribute equally to this work.
}

Correspondence to: Prof. Hong-Wei Zhang, Department of Epidemiology, Second Military Medical University, 800 Xiangyin Rd., Shanghai 200433, China. E-mail: hwzhang@smmu.edu.cn

How to cite this article: Tang WN, Ma LT, Deng Y, Wei Wang, Zhang HW. Efficacy and safety of immune checkpoint therapy in hepatocellular carcinoma: meta-analysis. Hepatoma Res 2019;5:19. http://dx.doi.org/10.20517/2394-5079.2019.07

Received: 22 Jan 2019 First Decision: 12 Mar 2019 Revised: 1 Apr 2019 Accepted: 4 Apr 2019 Published: 6 Jun 2019

Science Editor: Guang-Wen Cao Copy Editor: Cai-Hong Wang Production Editor: Huan-Liang Wu

\begin{abstract}
Aim: Immune checkpoint inhibitors (ICls) are proven to be an effective way to treat the disease of hematologic malignancies. But there is still plenty of uncertainty about the effectiveness of $\mathrm{ICls}$ on hepatocellular carcinoma. The Meta-analysis was conducted to evaluate the efficacy and safety of ICls treatment in patients with HCC.
\end{abstract}

Methods: Four electronic databases, including PubMed, Embase, Cochrane database, and ClinicalTrials.gov, were systematically retrieved for relevant observational studies published before November 1, 2018. The objective response rate (ORR) and adverse events were analyzed. Meta and Metafor Packages in R were utilized to accomplish meta proportion analysis.

Results: A total of 462 patients from 7 studies were included in this meta-analysis. The pooled estimated ORR of ICls was $19.8 \%$ (95\% $\mathrm{Cl} 16.4 \%$ to $23.7 \%$ ). No substantial heterogeneity was observed among studies $(Q=2.0427, P$ $\left.=0.92, l^{2}=0.0 \%\right)$. The common adverse events on any grade were saw in increased AST $(22.7 \%, 95 \% \mathrm{Cl} 13.8 \%$ to $35.2 \%$ ), fatigue $(20.9 \%, 95 \% \mathrm{Cl} 10.9 \%$ to $36.3 \%)$, rash $(18.5 \%, 95 \% \mathrm{Cl} 8.9 \%$ to $34.4 \%)$ and pruritus $(17.3 \%, 95 \% \mathrm{Cl}$ $13.5 \%$ to $21.8 \%$ ). Increased AST $(9.9 \%, 95 \% \mathrm{Cl} 4.4 \%$ to $21.0 \%$ ) and increased ALT (5.8\%, $95 \% \mathrm{Cl} 3.7 \%$ to $8.9 \%$ ) were the most common adverse events on grade greater than 3.

Conclusion: Although ICls treatment has a certain efficacy on liver cancer, it also causes some adverse events which should be noticed by clinicians.

Keywords: Hepatocellular, immune-checkpoint inhibitor, CLAT-4, PD1/PD-L1

\footnotetext{
(ㅇ) (1)

(C) The Author(s) 2019. Open Access This article is licensed under a Creative Commons Attribution 4.0 International License (https://creativecommons.org/licenses/by/4.0/), which permits unrestricted use, sharing, adaptation, distribution and reproduction in any medium or format, for any purpose, even commercially, as long as you give appropriate credit to the original author(s) and the source, provide a link to the Creative Commons license, and indicate if changes were made.
} 


\section{INTRODUCTION}

Liver cancer is the fourth most common cause of cancer-related death worldwide. Among all liver cancer type, hepatocellular carcinoma (HCC) is the most common neoplasm, accounting for approximately $90 \%$ cases $^{[1,2]}$. The common risk factors of HCC are cirrhosis, hepatitis B virus (HBV) or hepatitis C virus $(\mathrm{HCV})$ infection, alcohol abuse and metabolic syndrome ${ }^{[3]}$. The median overall survival of untreated HCC was 7 months, suggesting that its poor prognosis is attributable to advanced stages of diagnosis ${ }^{[4]}$. First line usage of multi-kinase inhibitor such as sorafenib was able to increase survival in advanced HCC from 7.9 months to 10.7 months (hazard ratio, 0.69). Unfortunately, this benefit was usually restricted by high resistance ${ }^{[5,6]}$. It's obviously that other approaches are still needed in treatment with advanced HCC. Immune checkpoints inhibitors (ICIs) therapy aiming to restore anticancer immunity has emerged as a promising therapy in liver cancer. Both clinical and preclinical studies revealed that there was a highly immunosuppressive tumor microenvironment and defective $\mathrm{T}$ cell recruitment in advanced $\mathrm{HCC}^{[7]}$. Exhaustion of $\mathrm{CD} 4^{+} \mathrm{T}$ cells has also been reported as a mechanism of immune evasion in $\mathrm{HCC}^{[8]}$. ICIs are monoclonal autoantibodies (mAbs) specifically targeting the inhibitory receptors on $\mathrm{T}$ cells (the so-called immune checkpoints). The most common types of ICIs are the cytotoxic T lymphocyte antigen 4 (CTLA4) and the programmed death 1 (PD-1) and its ligand PD-L1. Those all act as negative co-regulators to limit further T cell activation, which are normally responsible for limiting the escalated and chronic immune responses with deleterious autoimmune effects ${ }^{[9,10]}$. ICIs have been evaluated in a series of clinical trials for melanoma, non-small cell lung cancer (NSCLC) and renal cell carcinoma, and they have yielded favorable outcomes ${ }^{[11-13]}$. Some of the clinical trials with ICIs in liver cancers have been conducting in recent years, and more studies are still in the stage of recruiting. During the 2nd phase of clinical trials, ORR is an important outcome to evaluate the efficacy of anticancer drugs, which is also an essential factor to determine the carrying out of the 3rd phase of clinical trials. In this system review, we will retrieve studies about ICIs on liver cancer with outcome of ORR and analysis of the efficacy and safety.

\section{METHODS}

We carried out a comprehensive systematic search to identify studies about immune checkpoint inhibitor conducted on patients with HCC. The study was performed with adherence to PRISMA (Preferred Reporting Items for Systematic Reviews and Meta-Analysis) guidelines ${ }^{[14]}$.

\section{Literature search strategy}

We mainly searched four databases (PubMed, Embase, Cochrane database, and ClinicalTrials.gov) for articles published before November 1st, 2018. Controlled vocabulary and text word for synonymous terminology were both used in the search strategies. The following keywords were combined with Boolean logistical strategy for search: nivolumab, pembrolizumab, atezolizumab, avelumab, durvalumab, ipilimumab, tremelimumab, checkpoint inhibitors, $\mathrm{PD}$, programmed death 1, PD-L1, programmed cell death ligand 1, CTLA-4, cytotoxic T lymphocyte associate protein 4, and hepatocellular carcinoma, liver cancer, liver neoplasm, hepatic cancer, hepatic tumor.The search strategy in pubmed was as follow: (nivolumab OR pembrolizumab OR atezolizumab OR avelumab OR durvalumab OR ipilimumab OR tremelimumab OR "checkpoint inhibitors" OR "PD1" OR "programmed death 1" OR "PD-L1" OR "programmed cell death ligand1" OR "CTLA-4" OR "cytotoxic T lymphocyte associate protein 4") and ("hepatocellular carcinoma" OR "liver cancer" OR "liver neoplasm" OR "hepatic cancer" OR "hepatic tumor").

\section{Selection criteria}

Inclusion criteria was as follow: (1) randomized controlled clinical trials (RCTs) or non-randomized controlled clinical trials (n-RCTs); (2) patients pathologically diagnosed with hepatocellular carcinoma; (3) patients treated with PD1/PD-L1 or CTLA-4 monoclonal antibody; (4) studies with an outcome of objective response rate. Studies were excluded if they met the following criteria: (1) reviewer or case-report; (2) duplications with early publications from same authors or institutions; (3) unable to obtain full test. 


\section{Study selection}

Two investigators (Tang WN, Deng Y) independently screened the titles and abstracts of retrieved articles to choose potential relevant articles. Disagreement about particular studies were discussed and resolved by consensus.

\section{Data extraction}

Data extraction was carried out independently by the two reviewers (Tang WN, Deng Y). The following information was extracted from the eligible studies: information of the articles (author, published year, and study design), patient characteristics (number, age, area or nationality, race, and gender), liver disease condition (hepatitis virus infection, Eastern Cooperative Oncology Group (ECOG) performance scale, Child-Pugh stage, prior therapy), intervention in patients (agent, target, dosage, duration of dosing), outcome of efficacy (ORR).

\section{Statistical analysis}

The data analysis process was initially conducted by the third author (Ma LT). The pooled estimated ORRs and their 95\%CI were derived. Meta and Metafor Packages in R were utilized to accomplish meta proportion analysis. Logit transformation of raw proportion was performed before further analyses to increase validity. The ratio of between-study heterogeneity to total heterogeneity was quantified by $I^{2}$ and $P$ value. The assumption of homogeneity was considered invalid for $I^{2}>25 \%$ and $P<0.10$. A chi-square test (Q-test) was performed to test whether the heterogeneity between studies existed or not. If between-study heterogeneity were not significant, a fixed model would be applied to get a summarized proportion; otherwise a DerSimonian and Laired random effects model would be adopted. Two side $P<0.05$ was considered statistically significant.

\section{RESULTS}

\section{Eligible studies}

A total of 545 related articles were identified by the initial search strategy. After screening titles and abstracts, 525 studies were excluded because of irrelevant topics, review articles, molecular mechanism studies, experiments on animals, and clinical trials on recruiting. We then carefully reviewed the full texts of the remaining 20 potentially eligible papers. And then, 13 articles were excluded, because one of them was not able to obtain full test, two of them did not have the outcome of ORR, and ten papers were case-reports. Finally, seven studies were chosen for the following analysis. Figure 1 shows the study selection flowchart. Data from all eligible studies were obtained from published manuscripts.

\section{Study characteristics}

A total of 462 populations from seven studies were included in this meta-analysis. There was one paper published in 2013, five papers in 2017 and one paper in 2018. Six studies were carried out with multi-center clinical trials and one study was carried out with mono-center in China. Most of studies were in phase 1 or phase 2 of clinical trials. There were two studies conducting with CTLA-4 inhibitor (Tremelimumab), four studies with PD1/PD-L1 inhibitor (Pembrolizumab, Nivolumab, Durvalumab) and one study with combination of PD1 inhibitor and CTLA inhibitor (durvalumab and tremelimumab). Table 1 shows the main characteristics of the eligible trials.

\section{Efficacy of immune checkpoint inhibitors in HCC}

Overall, the pooled estimated ORR of patients treated with ICIs was 19.8\% (95\%CI: 16.4\%-23.7\%). No substantial heterogeneity was observed among single-study $\left(Q=2.0427, P=0.92, I^{2}=0.0 \%\right)$. Study of ElKhoueiry et al ${ }^{[18]}$ weighed most with estimated proportion of $19.6 \%$ (95\%CI: 14.8\%-25.5\%). And the second weighted article was Zhu et al. ${ }^{[17]}$ with estimated proportion of 17.3\% (95\%CI: 11.2\%-25.8\%). See Figure 2. 


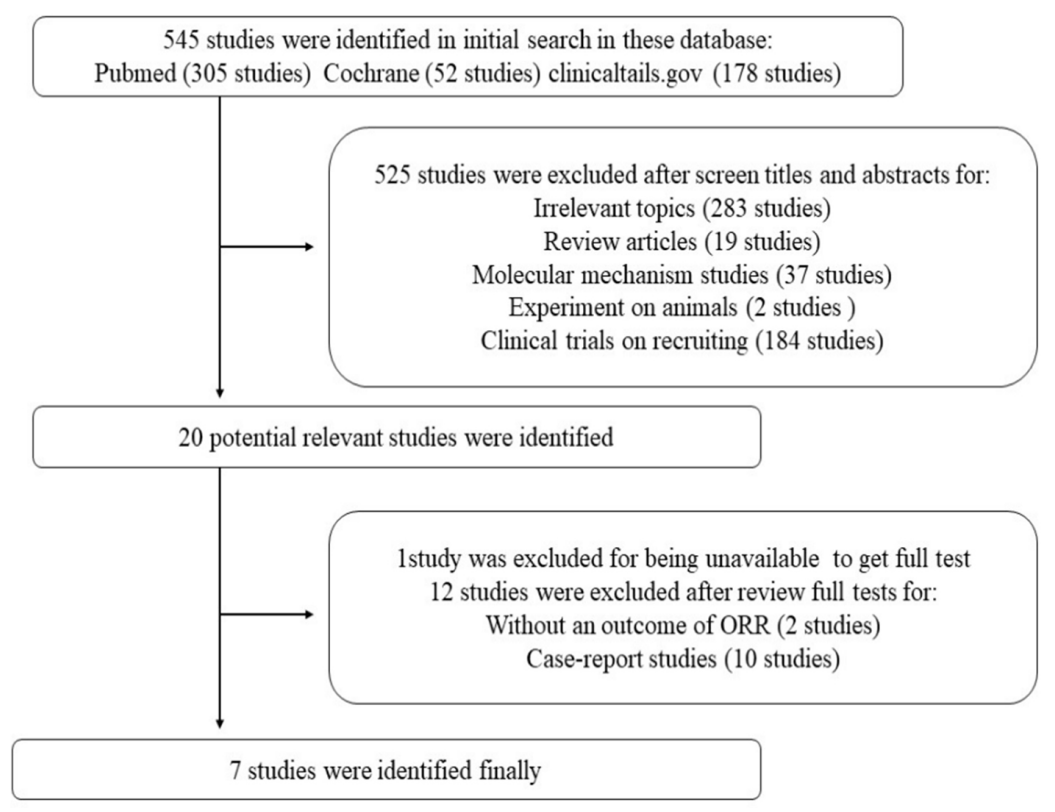

Figure 1. Flowchart program of selected studies

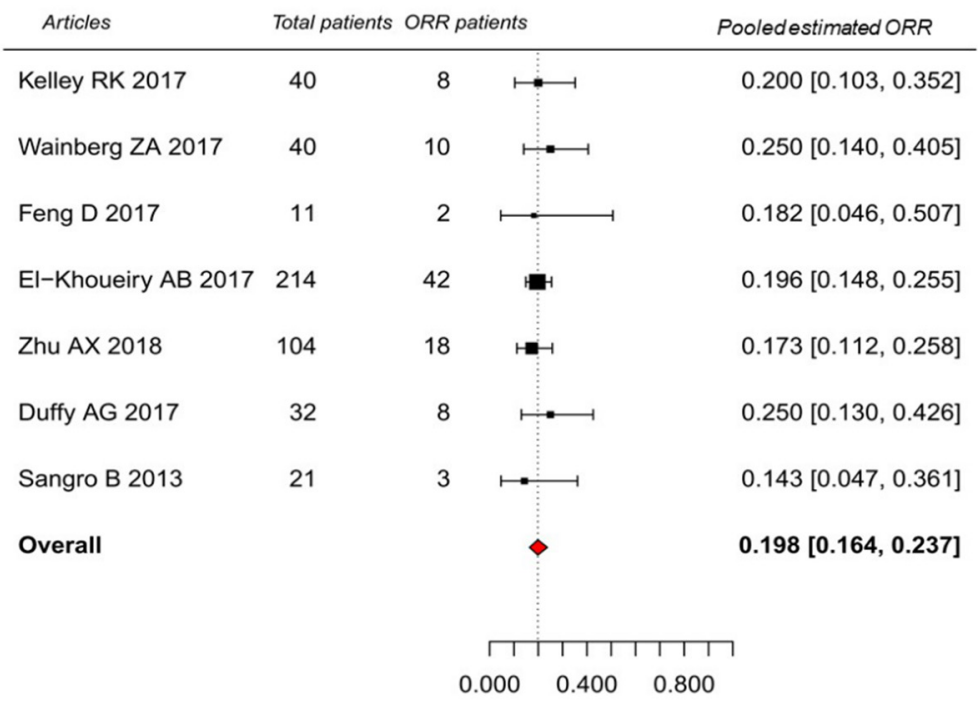

Figure 2. Objective response rate (ORR) of immune checkpoint inhibitor

\section{Publication bias}

A funnel plot was made to investigate potential publication bias. Unweighted Egger regression test was performed in each analysis to test whether the funnel plot is symmetrical or not [Figure 3].

\section{Adverse events of ICls}

If an adverse event was mentioned in more than 4 published papers, the incident rate of adverse event was estimated by R software. The pooled estimated incident rate and it's $95 \%$ confident interval were calculated on all grades and on grade greater than 3 respectively. The adverse events included in our study were fatigue, pruritus, rash, diarrhoea, nausea, asthenia, pulmonary toxicity, increased AST and increased ALT. The increased ALT was the most common adverse event whose pooled estimated incident rate was $22.7 \%$ (95\%CI: $13.8 \%-35.2 \%$ ), which was followed by fatigue $20.9 \%$ (95\%CI: $10.9 \%-36.3 \%$ ), rash $18.5 \%$ (95\%CI: 


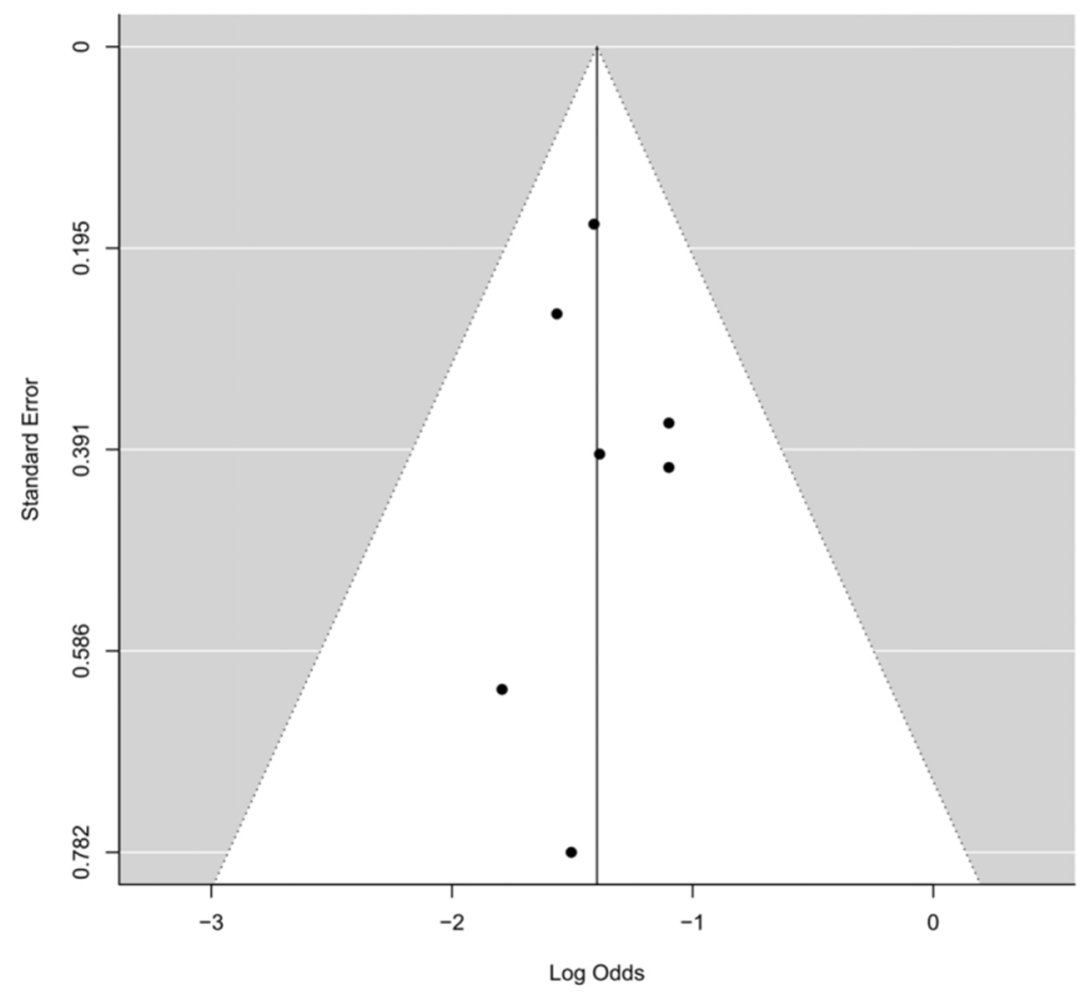

Figure 3. Publication biases of included references

8.9\%-34.4\%), and pruritus $17.3 \%$ (95\%CI: $13.5 \%-21.8 \%$ ). The most common adverse event on grade greater than 3 was increased AST, whose pooled estimated incident rate was $22.7 \%$ (95\%CI: $13.8 \%-35.2 \%$ ). The second was increased ALT 13.9\% (95\%CI: 8.8\%-21.3\%). The remaining adverse events of grade greater than 3, such as fatigue, pruritus, rash, diarrhoea, nausea, pulmonary toxicity, showed a small difference among pooled estimated incident rates, which were around $1 \%-2 \%$. The incident rate of Asthenia was only $0.9 \%$, as shown in Table 2.

\section{DISCUSSION}

This meta-analysis was performed to investigate the ORR published by paperswhich aimed to analyze the effectiveness of ICIs in patients with HCC. The derived overall estimated ORR reported on these nonheterogeneity papers is $19.8 \%$ (95\%CI: $16.4 \%-23.7 \%, P<0.001$ ). The result of this study is different from the investigation of other tumors. The difference maybe mainly caused by the heterogeneity among tumors. High response rate (50\%-90\%) of ICIs can be obtained with classical Hodgkin lymphoma, desmoplastic melanoma, Merkel cell carcinoma and microsatellite instability carcinoma. But the response rate of ICIs is reduced to $15 \%-25 \%$ when treating solid tumors such as non-small cell lung cancer, head and neck cancer, gastroesophageal cancer, bladder and urothelial cancer ${ }^{[22]}$. Compared to hematological malignancies, HCC as a solid tumor, shows not only a more complicated tumor microenvironment but the unique immune escape. All of these reasons may cause the low ORR in HCC patients treated with ICIs. One of the effective ways to enhance the drug response to ICIs is to obtain specific markers of liver cancer ${ }^{[23]}$. A high rate of ORR $(>30 \%)$ can be regard as a proper goal in the single arm clinical trial aiming at groundbreaking treatment ${ }^{[24]}$. There are currently five anti- PD-1/PD-L1 antibodies and two CTLA-4 blocking antibodies approved by the United States Food and Drug Administration (FDA). But only Nivolumab and Tremelimumab were approved to treat with HCC, with clinical trials of ICI agents currently ongoing ${ }^{[25]}$. The overall estimated ORR is only $19.8 \%$ based on the current study, which needs to be verified with multicenter randomized controlled studies 


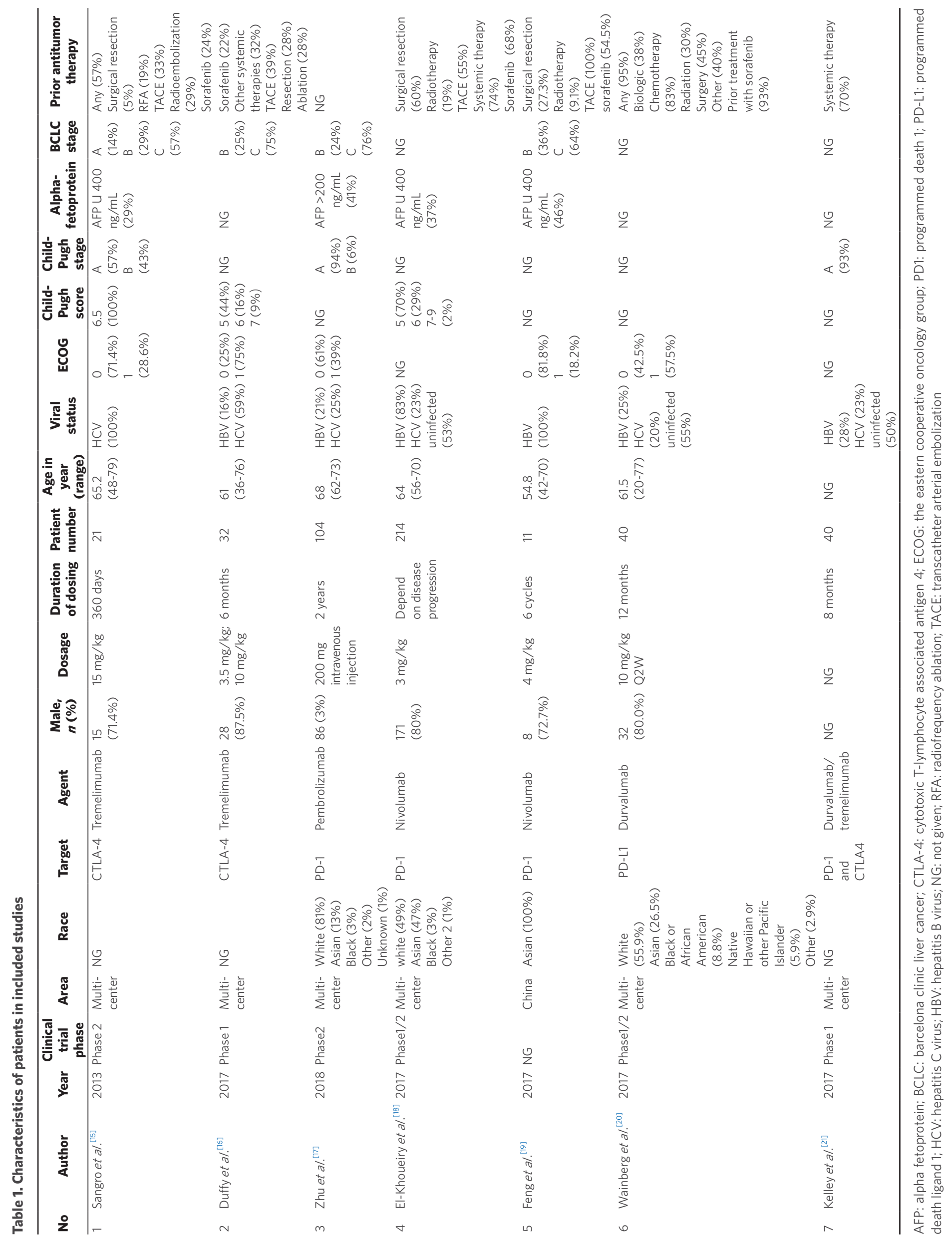


Table 2. The rate of major adverse events and their $95 \%$ confidence interval

\begin{tabular}{lccc}
\hline Adverse Events & Event rate on all grade (95\%Cl) & Ref. & Event rate on grade > 3 (95\%Cl) \\
\hline Fatigue & $20.8 \%(10.9 \%-36.3 \%)$ & {$[15,17-21]$} & $1.9 \%(0.9 \%-3.9 \%)$ \\
Pruritus & $17.3 \%(13.5 \%-21.8 \%)$ & {$[16-21]$} & $1.3 \%(0.4 \%-3.6 \%)$ \\
Rash & $18.5 \%(8.9 \%-34.4 \%)$ & {$[15-20]$} & $1.6 \%(0.5 \%-5.1 \%)$ \\
Diarrhoea & $12.5 \%(7.9 \%-19.1 \%)$ & {$[15,17-20]$} & $1.5 \%(0.3 \%-6.6 \%)$ \\
Nausea & $7.3 \%(5.0 \%-10.6 \%)$ & {$[15,17-19]$} & $1.8 \%(0.4 \%-8.3 \%)$ \\
Asthenia & $6.2 \%(4.1 \%-9.1 \%)$ & {$[17-20]$} & $0.9 \%(0.2 \%-3.4 \%)$ \\
Pulmonary toxicity & $3.3 \%(1.5 \%-6.8 \%)$ & {$[15-17,19-21]$} & $1.9 \%(0.7 \%-5.1 \%)$ \\
Increased AST & $22.7 \%(13.8 \%-35.2 \%)$ & {$[16-21]$} & $9.9 \%(4.4 \%-21.0 \%)$ \\
Increased ALT & $13.9 \%(8.8 \%-21.3 \%)$ & {$[16-20]$} & {$[15,17-19]$} \\
\hline
\end{tabular}

and clinical trials with other endpoints (such as overall survival, OS).

ICIs targeting CTLA-4 and PD-1/PDL-1 have dramatically changed the outcomes of patients with advancedstage malignancies. However, ICIs may cause unique side effects, known as immune-related adverse events (irAEs). These side effects are mostly transient and mild, but can occasionally be fatal. Our analysis indicated that the most common AEs associated with ICIs treatment in HCC patients was increased AST (22.73\%, 95\%CI: 13.8\%-35.2\%), which was also the most common AEs of grade greater than 3 (9.94\%, 95\%CI: 4.4\%-21.0\%). This result is inconsistent with previous studies on other cancers. Respectively, the most common AEs and severe AEs (grade 3-4) were fatigue in NSCLC ${ }^{[2,27]}$, fatigue and lipase elevation or fatigue and rash in melanoma ${ }^{[28,29]}$, low appetite and asthenia in urothelial carcinoma ${ }^{[30]}$, rash and lipase elevation in Hodgkin's lymphoma ${ }^{[31]}$, and neutropenia in lymphoma ${ }^{[32]}$. Patients with HCC treated with ICIs presented adverse event of fatigue in the second common place followed by rash and fatigue. Rash and fatigue are high incidence skin AEs. Skin AEs are the most original irAE, taking place every 3.6 weeks after treatment ${ }^{[33]}$. The pooled estimated incident rate of diarrhea is $12.46 \%$ (95\%CI: 7.9\%-19.1\%), which was the most common reported gastrointestinal toxicity. Other gastrointestinal toxicities such as abdominal pain, constipation, vomiting, were rarely reported and not taken into consideration here. Result from recent research showed that the incidence rate of diarrhea was higher with CTLA-4 blocked than the PD-1/PD-L1 blocked ${ }^{[34]}$. Adverse events of instance nausea, asthenia and pulmonary toxicity were less commonly reported in this study.

There are still some limitations in this study. Firstly, the final 7 studies included were all non-randomized controlled clinical trials; it might produce bias and downgrade the level of evidence. Secondly, some factors such as the origin of the HCC and patients' race might produce bias on outcomes, which can not be controlled in this meta analysis. However, the ORR is a straightforward index in evaluating the effectiveness of immunotherapy, and result from this meta analysis can be referred in clinical application.

\section{DECLARATIONS}

\section{Authors' contributions}

Conception and design: Zhang HW, Tang WN

Date analysis and interpretation: Tang WN, Ma LT, Deng Y, Wang W

Manucript preparation: Tang WN, Wang W

Critical revision and finalizing of the manuscript: Zhang HW

\section{Availability of data and materials}

Not applicable.

\section{Financial support and sponsorship}

This work was supported by National Key Basic Research Program of China (No. 2015CB554000).

\section{Conflicts of interest}

All authors declared that there are no conflicts of interest. 


\section{Ethical approval and consent to participate}

Not applicable.

\section{Consent for publication}

Not applicable.

\section{Copyright}

(c) The Author(s) 2019.

\section{REFERENCES}

1. Bray F, Ferlay J, Soerjomataram I, Siegel RL, Torre LA, et al. Global cancer statistics 2018: GLOBOCAN estimates of incidence and mortality worldwide for 36 cancers in 185 countries. CA Cancer J Clin 2018;68:394-424.

2. Llovet JM, Zucman-Rossi J, Pikarsky E, Sangro B, Schwartz M, et al. Hepatocellular carcinoma. Nat Rev Dis Primers 2016;2:16018.

3. Forner A, Llovet JM, Bruix J. Hepatocellular carcinoma. Lancet 2012;379:1245-55.

4. Giannini EG, Farinati F, Ciccarese F, Pecorelli A, Rapaccini GL, et al. Prognosis of untreated hepatocellular carcinoma. Hepatology 2015;61:184-90.

5. Cheng AL, Kang YK, Chen Z, Tsao CJ, Qin S, et al. Efficacy and safety of sorafenib in patients in the Asia-Pacific region with advanced hepatocellular carcinoma: a phase III randomised, double-blind, placebo-controlled trial. Lancet Oncol 2009;10:25-34.

6. Llovet JM, Ricci S, Mazzaferro V, Hilgard P, Gane E, et al. Sorafenib in advanced hepatocellular carcinoma. N Engl J Med 2008;359:378-90.

7. Zitvogel L, Tesniere A, Kroemer G. Cancer despite immunosurveillance: immunoselection and immunosubversion. Nat Rev Immunol 2006;6:715-27.

8. Chew V, Lai L, Pan L, Lim CJ, Li J, et al. Delineation of an immunosuppressive gradient in hepatocellular carcinoma using highdimensional proteomic and transcriptomic analyses. Proc Natl Acad Sci USA. 2017;114:E5900-E09.

9. Conway JR, Kofman E, Mo SS, Elmarakeby H, Van Allen E. Genomics of response to immune checkpoint therapies for cancer: implications for precision medicine. Genome Med 2018;10:93.

10. Ruggeri RM, Campenni A, Giuffrida G, Trimboli P, Giovanella L, et al. Endocrine and metabolic adverse effects of immune checkpoint inhibitors: an overview (what endocrinologists should know). J Endocrinol Invest 2018.

11. Wolchok JD, Chiarion-Sileni V, Gonzalez R, Rutkowski P, Grob JJ, et al. Overall survival with combined nivolumab and ipilimumab in advanced melanoma. N Engl J Med 2017;377:1345-56.

12. Horn L, Spigel DR, Vokes EE, Holgado E, Ready N, et al. Nivolumab Versus Docetaxel in Previously Treated Patients With Advanced Non-Small-Cell Lung Cancer: Two-Year Outcomes From Two Randomized, Open-Label, Phase III Trials (CheckMate 017 and CheckMate 057). J Clin Oncol 2017;35:3924-33.

13. Motzer RJ, Tannir NM, McDermott DF, Aren Frontera O, Melichar B, et al. Nivolumab plus ipilimumab versus sunitinib in advanced renal-cell carcinoma. N Engl J Med 2018;378:1277-90.

14. Moher D, Liberati A, Tetzlaff J, Altman DG, Group P. Preferred reporting items for systematic reviews and meta-analyses: the PRISMA statement. Ann Intern Med 2009;151:264-9, W64.

15. Sangro B, Gomez-Martin C, de la Mata M, Inarrairaegui M, Garralda E, et al. A clinical trial of CTLA-4 blockade with tremelimumab in patients with hepatocellular carcinoma and chronic hepatitis C. J Hepatol 2013;59:81-8.

16. Duffy AG, Ulahannan SV, Makorova-Rusher O, Rahma O, Wedemeyer H, et al. Tremelimumab in combination with ablation in patients with advanced hepatocellular carcinoma. J Hepatol 2017;66:545-51.

17. Zhu AX, Finn RS, Edeline J, Cattan S, Ogasawara S, et al. Pembrolizumab in patients with advanced hepatocellular carcinoma previously treated with sorafenib (KEYNOTE-224): a non-randomised, open-label phase 2 trial. Lancet Oncol 2018;19:940-52.

18. El-Khoueiry AB, Sangro B, Yau T, Crocenzi TS, Kudo M, et al. Nivolumab in patients with advanced hepatocellular carcinoma (CheckMate 040): an open-label, non-comparative, phase 1/2 dose escalation and expansion trial. Lancet 2017;389:2492-502.

19. Feng D, Hui X, Shi-Chun L, Yan-Hua B, Li C, et al. Initial experience of anti-PD1 therapy with nivolumab in advanced hepatocellular carcinoma. Oncotarget 2017;8:96649-55.

20. Wainberg ZA, Segal NH, Jaeger D, Lee K-H, Marshall J, et al. Safety and clinical activity of durvalumab monotherapy in patients with hepatocellular carcinoma (HCC). Journal of Clinical Oncology 2017;35:4071.

21. Kelley RK, Abou-Alfa GK, Bendell JC, Kim T-Y, Borad MJ, et al. Phase I/II study of durvalumab and tremelimumab in patients with unresectable hepatocellular carcinoma (HCC): phase I safety and efficacy analyses. Journal of Clinical Oncology 2017;35:4073.

22. Ribas A, Wolchok JD. Cancer immunotherapy using checkpoint blockade. Science 2018;359:1350-5.

23. Clark DP. Biomarkers for immune checkpoint inhibitors: the importance of tumor topography and the challenges to cytopathology. Cancer Cytopathol 2018;126:11-9.

24. Oxnard GR, Wilcox KH, Gonen M, Polotsky M, Hirsch BR, et al. Response rate as a regulatory end point in single-arm studies of advanced solid tumors. JAMA Oncol 2016;2:772-9.

25. Xu F, Jin T, Zhu Y, Dai C. Immune checkpoint therapy in liver cancer. J Exp Clin Cancer Res 2018;37:110.

26. Planchard D, Yokoi T, McCleod MJ, Fischer JR, Kim YC, et al. A phase III study of durvalumab (MEDI4736) with or without tremelimumab for previously treated patients with advanced NSCLC: rationale and protocol design of the ARCTIC study. Clin Lung Cancer 2016;17:232-6.e1. 
27. Gettinger SN, Horn L, Gandhi L, Spigel DR, Antonia SJ, et al. Overall survival and long-term safety of nivolumab (Anti-programmed death 1 antibody, BMS-936558, ONO-4538) in patients with previously treated advanced non-small-cell lung cancer. J Clin Oncol 2015;33:2004-12.

28. Weber JS, D'Angelo SP, Minor D, Hodi FS, Gutzmer R, et al. Nivolumab versus chemotherapy in patients with advanced melanoma who progressed after anti-CTLA-4 treatment (CheckMate 037): a randomised, controlled, open-label, phase 3 trial. Lancet Oncol 2015; $16: 375-84$.

29. Hamid O, Robert C, Daud A, Hodi FS, Hwu WJ, et al. Safety and tumor responses with lambrolizumab (anti-PD-1) in melanoma. N Engl J Med 2013;369:134-44.

30. Powles T, Eder JP, Fine GD, Braiteh FS, Loriot Y, et al. MPDL3280A (anti-PD-L1) treatment leads to clinical activity in metastatic bladder cancer. Nature. 2014;515:558-62.

31. Ansell SM, Lesokhin AM, Borrello I, Halwani A, Scott EC, et al. PD-1 blockade with nivolumab in relapsed or refractory Hodgkin's lymphoma. N Engl J Med 2015;372:311-9.

32. Armand P, Nagler A, Weller EA, Devine SM, Avigan DE, et al. Disabling immune tolerance by programmed death-1 blockade with pidilizumab after autologous hematopoietic stem-cell transplantation for diffuse large B-cell lymphoma: results of an international phase II trial. J Clin Oncol 2013;31:4199-206.

33. Weber JS, Kahler KC, Hauschild A. Management of immune-related adverse events and kinetics of response with ipilimumab. J Clin Oncol 2012;30:2691-7.

34. Callahan MK, Wolchok JD. At the bedside: CTLA-4- and PD-1-blocking antibodies in cancer immunotherapy. J Leukoc Biol 2013;94:41-53. 